\title{
A Case of Metastatic Malignant Melanoma Simulating Granuloma Annulare
}

\author{
Haley D. Heibel, MD, ${ }^{1}$ Parneet Dhaliwal, DO, ${ }^{2}$ Etan Marks, DO, ${ }^{3}$ Clay J. Cockerell, MD, MBA ${ }^{1,4}$ \\ ${ }^{1}$ Cockerell Dermatopathology, Dallas, TX \\ 2Department of Pathology, Baylor University Medical Center, Dallas, TX \\ ${ }^{3}$ Advanced Dermatology and Cosmetic Surgery, Delray Beach, FL \\ ${ }^{4}$ Departments of Dermatology and Pathology, UT Southwestern Medical Center, Dallas, TX
}

\section{ABSTRACT}

Malignant melanoma and particularly metastatic melanoma represent a diagnostic challenge due to the wide variety of histologic patterns, resemblance to benign entities, and extensive range of clinical presentations. A high index of suspicion for melanoma is important for accurate diagnosis, especially when there is a previous history of malignancy. Here, we present a patient with a history of melanoma and locally metastatic melanoma, who subsequently developed a nodule on his right forearm near the site of his previous melanoma excision. Histologically, the melanoma appeared as granuloma annulare (GA) with benign cytologic features, but was identified as metastatic melanoma using SOX-10 immunohistochemical staining. Other malignancies, including lymphomas, leukemias, sarcomas, and cutaneous metastases of internal malignancies, have mimicked GA and interstitial granulomatous processes. Therefore, further immunohistochemical staining should be performed to assess for metastatic disease in the setting of a histological pattern that resembles a benign granulomatous process in a patient with a history of malignancy, including malignant melanoma.

\section{INTRODUCTION}

Malignant melanoma is a complex malignancy that can present with a wide range of histological patterns, including mimicking other malignant tumors and benign entities, such as interstitial granulomatous processes. ${ }^{1,2}$ Similarly, metastatic melanoma has a wide variety of clinical presentations that may not be visible to the clinician and may not seem suspicious for melanoma. ${ }^{1}$ All of these factors contribute to the challenges with making an accurate diagnosis of metastatic melanoma. Beyond the classical forms of melanoma (lentigo maligna, superficial spreading, nodular, acral-lentiginous, and mucosal), there are other unusual variants. ${ }^{2}$ These variants may be amelanotic and mimic other benign and malignant processes both clinically and histopathologically, often require additional testing for diagnosis, and represent a diagnostic challenge. ${ }^{3}$

In addition, granulomatous reactions associated with melanoma, cutaneous granulomatous reactions following the initiation of treatment for metastatic melanoma, and metastatic melanoma mimicking an interstitial granulomatous reaction have been reported. ${ }^{1,4-8}$ Here, we present a case of a patient with a history of melanoma and locally metastatic melanoma, who developed a right forearm nodule near the previous melanoma excision site. Histologically, the lesion presented as

May 2021 Volume 5 Issue 3 


\section{SKIN}

granuloma annulare (GA) with benign cytologic features, but was identified as metastatic melanoma through positive immunohistochemical staining for SOX-10.

\section{CASE PRESENTATION}

An 89-year-old male with a history of melanoma presented with a flesh-colored nodule on the right dorsal forearm adjacent to the site of the previous melanoma excision (Figure 1). His primary melanoma had been diagnosed 14 years previously on his right forearm with an initial depth of 1.4 $\mathrm{mm}$ (pathologic stage: pT2a). He additionally had 3 locally metastatic melanomas near the surgical excision site of the original melanoma occurring 11,5 , and 1 year(s) prior to presentation. Sentinel lymph node biopsies were negative for the primary melanoma and the first locally metastatic melanoma, but there was no sentinel lymph node biopsy done for the second locally metastatic melanoma. There was no evidence of other metastases at the time of evaluation of the first and second locally metastatic melanomas by PET/CT imaging of the head, neck, chest, abdomen, and pelvis. The patient then underwent excision of these lesions with negative margins. However, the second metastatic lesion required re-excision for a close margin of 1 $\mathrm{mm}$.

When the third locally metastatic melanoma developed on his right forearm, he again underwent excision of this lesion. At this time, a PET/CT scan demonstrated an enlarged $1.6 \mathrm{~cm}$ right sided pelvic lymph node, although CT-guided FNA of the right pelvic lymph node was nonspecific. A repeat core needle biopsy of the lymph node 2 months later showed no evidence of malignancy. However, another PET-CT scan 6 months later showed a metabolically

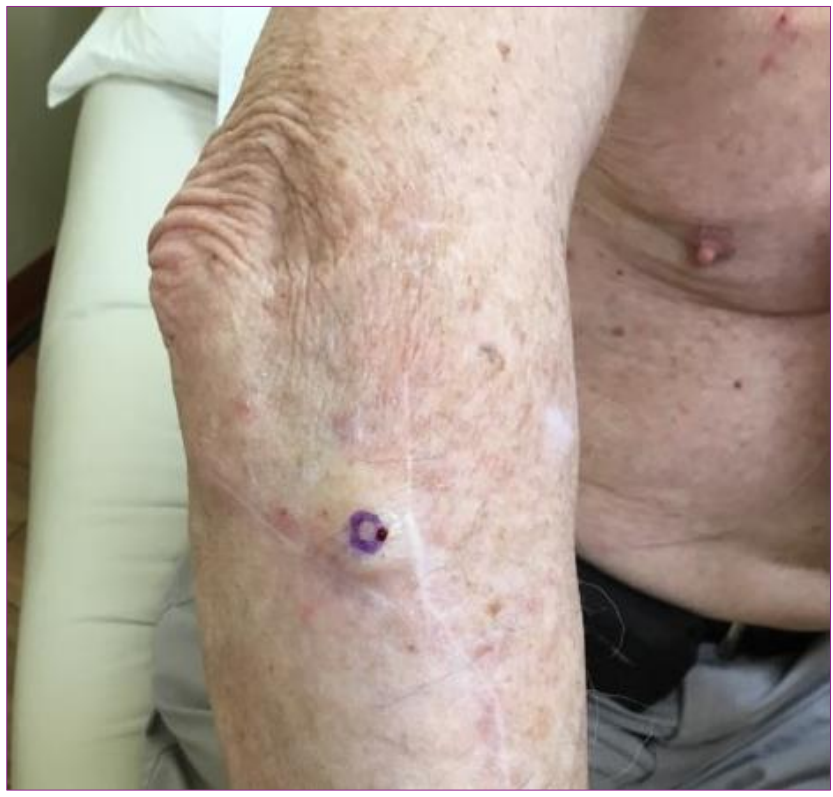

Figure 1. Clinical presentation: A flesh-colored nodule on the right dorsal forearm adjacent to the site of previous melanoma excision

active right adrenal mass and right external iliac lymph node. CT guided biopsy of the right adrenal mass was consistent with metastatic melanoma. Genetic testing was negative for BRAF mutation. He was initiated on nivolumab (240 mg every 2 weeks) 10 months subsequent to the diagnosis of this metastatic melanoma and 2 months prior to the development of this most recent skin lesion.

Initial histopathologic examination of the most recent skin lesion (fourth lesion appearing at the site of the primary melanoma excision) demonstrated discrete areas of what appeared to be palisading histiocytes surrounding collections of mucin and perivascular lymphocytes with benign appearing cytologic features consistent with probable GA (Figure 2). Given the patient's history of melanoma, immunohistochemical staining was performed to exclude a melanocytic process and revealed cells strongly positive for SOX-10 (Figure 3). However, only scattered cells were highlighted with CD68. These features were 
consistent with metastatic melanoma simulating GA.

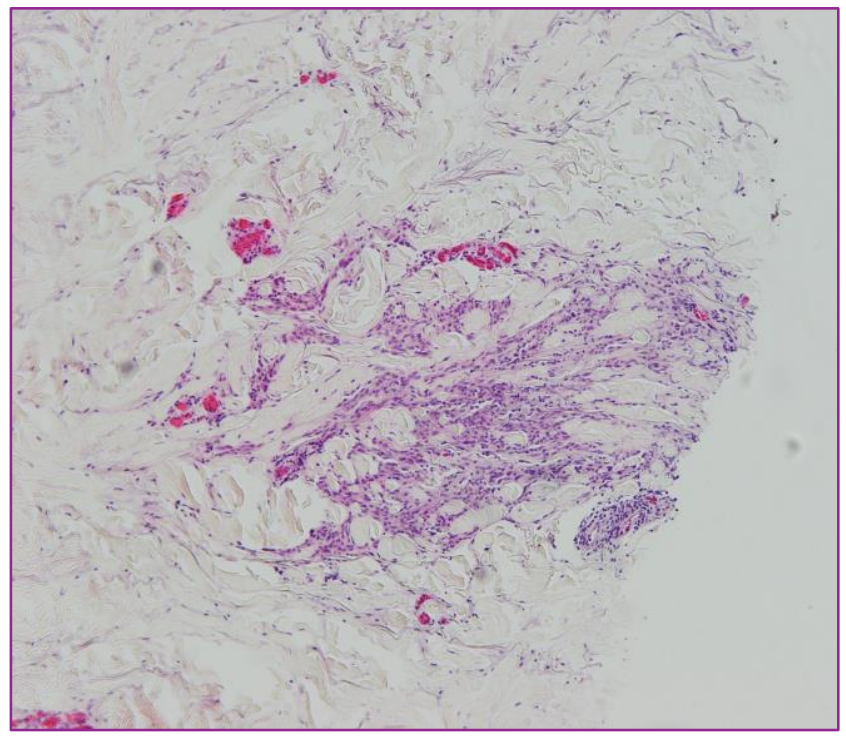

Figure 2. Histologic images showing an interstitial infiltrate resembling granuloma annulare with cells wrapping around collagen bundles. The cells have a benign appearance without any obvious mitotic figures or significant pleomorphism.

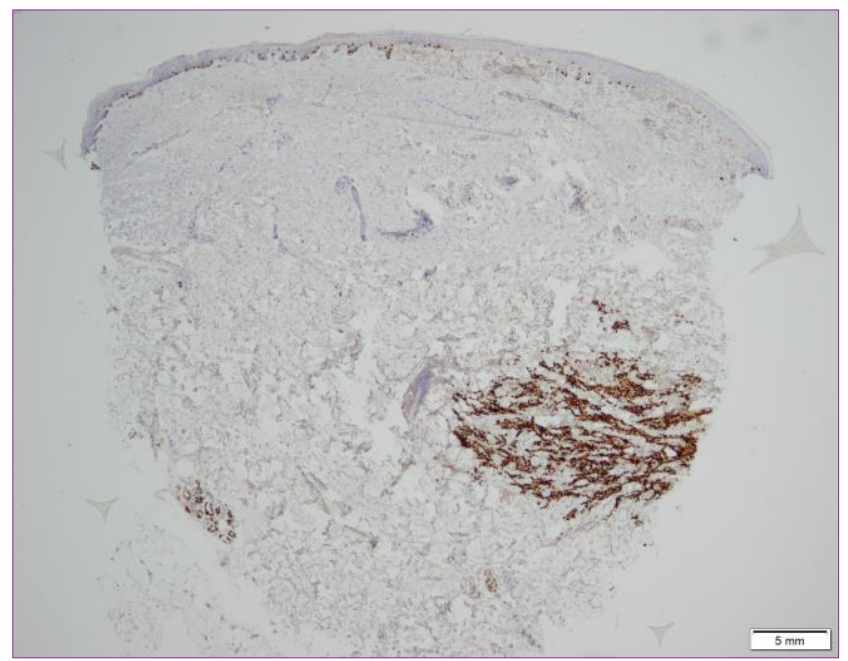

Figure 3. SOX-10 immunohistochemical staining revealing the cells are melanocytes.

The patient has continued nivolumab therapy (240 $\mathrm{mg}$ every 2 weeks) to the present time. He also completed radiation therapy for his right adrenal lesion. He has been followed with serial PET/CT scans, and a slight increase in the size of the right adrenal lesion was observed 14 months after its initial identification, but no other evidence of metastases or disease progression has been identified.

\section{DISCUSSION}

Cutaneous manifestations of lymphomas (including mycosis fungoides), leukemias, and sarcomas have resembled GA both clinically and histologically. ${ }^{1,9,10}$ Hartman et $\mathrm{al}^{9}$ reported 3 cases of cutaneous metastases of internal malignancies (including breast and salivary gland mucoepidermoid carcinomas) that histologically mimicked interstitial granulomatous processes, with one case clinically appearing as GA. The malignant carcinoma cells were organized in an interstitial pattern, although some of the cells did not have significant cellular atypia.

Parekh et al ${ }^{1}$ described another patient who presented with a melanoma and subsequently developed cutaneous metastases. Wide excision of the scar and metastases demonstrated histologic findings of an amelanotic process separate from the original wound of operation with a differential diagnosis of GA and some other form of an interstitial granulomatous process. The amelanotic, poorly differentiated cells were identified as metastatic melanoma through positive MART-1 immunohistochemical staining.

The etiology of a granulomatous process associated with metastatic melanoma is unclear, but theories include a cell-mediated immune response against an antigenic factor derived from tumor cells or that melanoma cells secrete cytokines which attract histiocytes and lead to granuloma formation. ${ }^{7}$ In the setting of granulomatous reactions occurring during the treatment of melanoma, Park et $\mathrm{al}^{6}$ suggested this may represent immune activation against tumor

May 2021 Volume 5 Issue 3 
cells. Reports of GA occurring subsequent to the initiation of immune checkpoint inhibitors have been reported as well. ${ }^{8}$ Our patient had initiated nivolumab 2 months prior to his presentation with this new skin lesion. Therefore, the histiocytes admixed with the melanocytes could be related to some form of immune activation and gave the appearance of GA.

\section{CONCLUSION}

Metastatic melanoma can present with a wide variety of clinical presentations. ${ }^{1}$ This case illustrates the importance of the provision of accurate and complete medical history by the submitting clinician and communicating this information clearly to the dermatopathologist. This information may affect stains selected by the dermatopathologist and the histopathologic diagnosis, especially when the histopathologic pattern is a granulomatous process in a patient with a history of malignancy. In this case, clinical history of a melanoma was critical for achieving an accurate histopathologic diagnosis because it prompted further staining to assess for a melanocytic process. In the presence of a histological pattern that resembles a benign granulomatous process in a patient with a history of malignancy, including malignant melanoma, further immunohistochemical staining should be performed to assess for metastatic disease. $^{9}$

\section{Abbreviations used: Granuloma annulare (GA)}

Acknowledgements: The authors would like to thank Andrew Ondo, M.D., for providing the clinical information and clinical photo for this manuscript.

Conflict of Interest Disclosures: Haley D. Heibel, Parneet Dhaliwal, Etan Marks, and Clay J. Cockerell declare that they have no conflicts of interests that may be related to the contents of this manuscript.
Funding: None

Corresponding Author:

Haley D. Heibel, MD

2110 Research Row, Suite 100

Dallas, TX 75235

Phone: 214-530-5200

Email: hheibel@dermpath.com

\section{References:}

1. Parekh V, Miller CJ, Elenitsas R, Chu EY. Cutaneous metastases of melanoma mimicking interstitial granulomatous processes. Am J Dermatopathol. 2018;40(9):706-707.

2. Rongioletti F, Smoller BR. Unusual histological variants of cutaneous malignant melanoma with some clinical and possible prognostic correlations. J Cutan Pathol. 2005;32(9):589-603.

3. Barnhill RL, Gupta K. Unusual variants of malignant melanoma. Clin Dermatol. 2009;27(6):564-587.

4. Garrido MC, Gutierrez C, Riveiro-Falkenbach E, Ortiz P, Rodriguez-Peralto JL. BRAF inhibitor-induced antitumoral granulomatous dermatitis eruption in advanced melanoma. Am J Dermatopathol. 2015;37(10):795-798.

5. Korman AM, Nisar MS, Somach SC. Subclinical granulomas in benign skin lesions heralding the onset of BRAF and MEK inhibitor-associated granulomatous dermatitis in a patient with metastatic melanoma. JAAD Case Reports. 2018;4(7):722-724.

6. Park JJ, Hawryluk EB, Tahan SR, Flaherty K, Kim CC. Cutaneous granulomatous eruption and successful response to potent topical steroids in patients undergoing targeted BRAF inhibitor treatment for metastatic melanoma. JAMA Dermatol. 2014;150(3):307-311.

7. Robert C, Schoenlaub P, Avril M, et al. Malignant melanoma and granulomatosis. Br J Dermatol. 1997;137(5):787-792.

8. Wu J, Kwong BY, Martires KJ, et al. Granuloma annulare associated with immune checkpoint inhibitors. J Eur Acad Dermatol Venereol. 2018;32(4):e124-e126.

9. Hartman RI, Chu EY, Acker SM, James WD, Elenitsas $\mathrm{R}$, Kovarik CL. Cutaneous metastases from visceral malignancies mimicking interstitial granulomatous processes: A report of 3 cases. Am J Dermatopathol. 2013;35(5):601-605.

10. Jouary T, Beylot-Barry M, Vergier B, Paroissien J, Doutre MS, Beylot C. Mycosis fungoides mimicking granuloma annulare. Br J Dermatol. 2002;146(6):11021103. 